\title{
Title:
}

\section{PROSPECTIVE STUDY OF THE UTILITY OF COMPUTED TOMOGRAPHY TRIAGE OF PLEURAL BIOPSY STRATEGIES IN PATIENTS WITH PLEURAL DISEASES}

\section{Authors:}

Muzaffer Metintas, Prof.,MD

Eskisehir Osmangazi University Lung and Pleural Cancers Research and Clinical Center, Medical Faculty Department of Chest Diseases, Eskisehir, Turkey,

muzaffermetintas@gmail.com

Güntülü Ak, Assoc.Prof., MD, PhD

Eskisehir Osmangazi University Lung and Pleural Cancers Research and Clinical Center, Medical Faculty Department of Chest Diseases, Eskisehir, Turkey, guntuluak@gmail.com

Selma Metintas, Prof.,MD, PhD

Eskisehir Osmangazi University Lung and Pleural Cancers Research and Clinical Center, Medical Faculty Department of Public Health, Eskisehir, Turkey,

selmametintas@hotmail.com

Huseyin Yıldırım, Prof.,MD

Eskisehir Osmangazi University Medical Faculty Department of Chest Diseases, Eskisehir, Turkey, heylu12002@yahoo.com

Emine Dündar, Prof., MD

Eskisehir Osmangazi University Lung and Pleural Cancers Research and Clinical Center, Medical Faculty Department of Pathology, Eskisehir, Turkey, edundar@ogu.edu.tr

Najib Rahman, Prof., MD

Nuffield Department of Medicine, Oxford Respiratory Trials Unit, University of Oxford, UK najib.rahman@ndm.ox.ace.uk

Corresponding Author: Muzaffer Metintas

Eskisehir Osmangazi University Lung and Pleural Cancers Research and Clinical Center, Medical Faculty Department of Chest Diseases, Eskisehir, Turkey

muzaffermetintas@gmail.com, Phone: +90 2222392979 Ex.7050 Fax:+90 2222394714

Running head: Pleural biopsy strategies

\section{Authorship statement:}

M. Metintas, G. Ak, and S Metintas had the idea for and designed the study. Abrams' pleural biopsy procedures were done by G. Ak, H. Yildırım, M. Metintas. Statistical tests were performed by S. Metintas. The report was drafted by M. Metintas, S. Metintas, and N. Rahman and was edited by M. Metintas and N. Rahman. Thoracoscopy was performed by M. Metintas with G. Ak or with H. Yıldırım. Histopathological studies on the biopsy samples of the patients were performed by E. Dundar. The management of the patients in clinic were performed by G. Ak, H. Yildırım, M. Metintas.

\section{Disclosure statement:}

All authors in this paper have no financial or personal conflicts of interest to disclose. June 13, 2018. 


\begin{abstract}
Background: This study aimed to prospectively evaluate the efficacy and reliability of a diagnostic workup, triaging pleural biopsy method according to baseline computerized tomography (CT) findings in the diagnosis of pleural diseases.
\end{abstract}

Methods: Patients with pleural pathology were divided into three arms according to findings on CT scans. Arm A: Subjects with pleural thickening/lesion in addition to pleural effusion. These patients underwent CT- scan guided Abrams' needle pleural biopsy (CT-ANPB). Arm B: Subjects with pleural effusion alone or suspected benign asbestos pleurisy (BAP). This group underwent medical thoracoscopy (MT). Arm C: Subjects with only pleural thickening. This group underwent ultrasonography guided cutting needle pleural biopsy (US-CNPB). MT was planned in patients who did not have specific diagnosis in CT-ANPB group. When patients with a histopathological diagnosis of fibrinous pleuritis after MT were assessed in terms of risk factors for malignant pleural diseases, we offered further invasive procedure.

Results: A total of 164 patients were enrolled to the study. Diagnostic sensitivity after initial procedure were 90.2\% in Arm A, 93.3\% in Arm B, 95.2\% in Arm C, and 92.4\% in entire workup. Negative predictive value of entire work up was $90.4 \%$ for malignant pleural mesothelioma, $97.1 \%$ for metastatic malignant pleural diseases and $100 \%$ for tuberculous pleurisy. Five cases who had a diagnosis of fibrinous pleuritis after MT were detected to have risk factors, four of which (80\%) indicated malignant disease. Complication rates were low and acceptable.

Conclusion: Using CT scans to triage appropriate pleural biopsy method is associated with high diagnostic success. We recommend the proposed diagnostic workup in this study may be used as a diagnostic algorithm for pleural diseases which require histopathological analysis. Determination of risk factors predicting malignant disease in patients where fibrinous pleuritis is reported after MT would be useful for clinical practice.

Key words: Pleural effusion, Computed tomography, Medical thoracoscopy, Pleural biopsy, Diagnosis 


\section{INTRODUCTION}

In the presence of an undiagnosed effusion after initial pleural aspirate, medical thoracoscopic and image-guided pleural biopsies under computerized tomography (CT) or ultrasonography (US) with Abrams' needle or cutting needles are the preferred diagnostic modalities, as both are characterized by a greater diagnostic sensitivity compared with blind pleural biopsy for the diagnosis of pleural disease (1-9). The choice between medical thoracoscopy (MT) or image guided biopsies is influenced by physician preference, expertise, availability of instruments, cost, and visualizable of pleural lesions (3,10-16). In some patients, as pleural lesions or thickening cannot be clearly located, the benefit of CT or US guided biopsy may be reduced $(9,10)$. Additionally, in some benign pleural diseases such as benign asbestos pleurisy (BAP), the pleural space needs to be examined to rule out malignant pleural involvement, where MT becomes the preferred modality for diagnosis $(3,9,12,17)$. In this respect, there is no widely accepted algorithm to select biopsy method for patients with distinctive pleural characteristics.

It is well established that $\mathrm{CT}$ is indicated in patients in whom pleural invasive procedures are considered, and the abilities of pulmonologists regarding CT assessment are regarded as relatively high. Therefore, CT scans may be used at either the bedside or at endoscopy to guide pleural procedures without requiring any further imaging modality. Here, we designed and tested a diagnostic workup to determine the most appropriate pleural biopsy method, individualized according to initial CT findings.

Based on evidence from recent articles, reviews (2,6,8,14,17-22) and findings from our own research $(9,10,23)$, a specific diagnostic algorithm was conceived. The algorithm involved selection of pleural biopsy method according to initial CT findings as follows:

- In cases of cytology negative pleural effusion associated with pleural thickening/lesions seen on the diagnostic CT, CT-guided Abrams needle pleural biopsy (CT-ANPB) was conducted.

- In cases with fluid only on CT, when BAP was suspected or the lesion was inaccessible by Abrams' needle, MT was used as the primary diagnostic method utilized (9).

- In cases of isolated pleural thickening with no pleural effusion, or a small effusion for which Abrams' was not feasible, US- guided cutting needle pleural biopsy (US-CNPB) was used. 
In this study we aimed to prospectively evaluate the efficacy and reliability of this diagnostic workup, triaging biopsy method according to baseline CT.

\section{METHOD and MATERIALS}

This study was conducted in the Chest Diseases Department of Eskisehir Osmangazi University from December 2010 to June 2014. The study was approved by the Ethical Committee of Eskisehir Osmangazi University (PR-11-01-31-21/2011.8.02). The clinical approval number (ClinicalTrials.gov Identifier) was NCT03153501.

\section{Patients:}

Patients with the following criteria were invited to participate in the study:

- Evidence of pleural diseases with or without pleural effusion, for which a specific diagnosis could not be determined by initial thoracocentesis and/or clinical and radiological findings

- Willingness to participate in the study and undergo an invasive procedure

- Those not included in another clinical trial of diagnostic strategy diagnosis.

Exclusion criteria included the following

- Age under 18 years

- Presence of parapneumonic effusion

- Presence of a contraindication to pleural biopsy

- The presence of any other systemic disease which could affect CT or US assessments.

Patients were thoroughly informed of the study procedures before being taken into the study, and written consent was requested.

\section{Diagnostic strategy:}

The flow-chart applied for diagnostic workup used in this study is shown at Figure 1

\section{Figure 1}

Figure 1. Diagnostic flow-chart.

Patients with pleural pathology were divided into three arms according to findings on CT scan: Arm A: Subjects with pleural thickening/lesion in addition to pleural effusion. Arm B: Subjects with pleural effusion alone or pleural lesion at an atypical location for Abrams' or cutting needle 
or those with suspected BAP upon epidemiological, clinical, and thoracentesis properties (defined as patients with definite asbestos exposure and whose clinical and radiologically findings required the exclusion of BAP from the differential diagnosis). Arm C: Subjects with pleural thickening alone or mild encysted pleural effusion in CT scans, and findings was required to be examined histopathologically. All procedures were performed in the in-patient setting.

For patients who did not have a specific malignant or tuberculous diagnosis upon CT-ANPB in Arm A or C, MT was offered and alternative invasive methods (such as surgery) were discussed. At the patient's discretion, video-assisted thoracoscopic surgery (VATS), open pleural biopsy, or an observational strategy without any procedure were offered.

When pleural biopsy results suggested "fibrinous pleuritis" in patients undergoing MT, patients were assessed for the presence of risk factors for possible malignant pleural involvement. These factors were as follows (23): Thoracoscopist's macroscopic impression of a non-benign condition , CT findings suggestive of malignant pleural change, or the presence of pain on the effected side. The criteria for malignant pleural involvement on CT were one or more of the following findings:

- Circumferential plerual thickneing

- Mediastinal pleural involvement

- Pleural nodularity

- Pleural thickness more than $1 \mathrm{~cm}$

- Invasion of thoracic structures such as pericardium, chest wall, diaphragm, mediastinum

- Nodular involvement of fissures $(16,23)$.

Any patient with at least one of these risk factors was offered VATS or open surgical biopsy. Either of these methods were also offered to those who had fibrinous pleuritis according on USCNPB. CT-ANPBs and US-CNPB were performed as previously described (8-11).

\section{CT scan-guided Abrams needle pleural biopsy:}

Contrast enhancemed CT of all patients was performed. The thorax was scanned at a thickness of $5 \mathrm{~mm}$ from the apex of the lungs to the costophrenic recess. $80 \mathrm{~mL}$ of non-ionic contrast substance was administered through an arm vein, and the scan was initiated 30 seconds following the infusion of the contrast medium.

Abrams' biopsy procedures were performed with the entry site determined by CT-scan, as the most suitable and accessible lesion by reviewing CT scans on monitors while patients were in the procedure room. The distance between the entry site and the target point was measured two- 
dimensionally on CT. After taking measurements as described, the entry site for Abrams' needle was marked on the skin of the patient at the corresponding point of the lesion on the CT-scan. Abrams' needle biopsies were performed according to standardized procedure (8-10). Four to six biopsy specimens were taken from the parietal pleura using the distal tip of the Abrams' needle at a $45^{\circ}$ angle to avoid causing lacerations of the intercostal vessels through the same entry point.

\section{US-guided cutting needle pleural biopsy:}

Transthoracic US was performed in the procedure room. The preferred patient position for the procedure was sitting, with the subject's arms folded across the chest and supported by a table. Surveillance of the dorsolateral thoracic wall was performed using a standard $3.75 \mathrm{MHz}$ curvilinear probe. Cutting needle biopsies were performed using manually operated 14-gauge TruCut biopsy needles. The biopsy site was determined by US including depth of penetration. The skin was marked and the biopsy performed while the patients remained in the same position $(8,9,11,18)$. Four to six biopsy specimens were taken from the parietal pleura.

MT was done with a rigid thoracoscope (Karl Storz, Germany) under mild sedation and local anesthesia. At least six biopsy specimens were taken from abnormal sites of parietal pleura at thoracoscopy (10).

Following each procedure, the biopsy specimens were immediately fixed in formalin and sent to the pathology department for histopathological analysis. If suspicion of tuberculous pleurisy was high, an additional biopsy specimen was sent to the laboratory in an isotonic saline solution for bacteriologic investigation, including for Mycobacterium tuberculosis.

Patients with benign pleural disease were followed for a minimum follow-up of 36 months to ensure they did not develop a malignant pleural disease.

\section{Statistical Analysis:}

The primary end point of this study was the determination of both the sensitivity and the complication rates of the invasive methods with respect to the diagnosis of pleural disease. SPSS, version 15.0 software (SPSS Inc. Chicago, Illinois) was used for the statistical analysis. The complication rate for both methods were calculated by Post hoc Power analysis.

\section{RESULTS}


The number of subjects enrolled to the study upon having pleural pathology according to the inclusion criteria was 169 . However, five were excluded after initial evaluation or due to being lost to follow-up after procedure. The distribution of the remaining 164 subjects by age, gender, and initial pleural biopsy method is shown in Table 1 .

\section{$\underline{\text { Table } 1}$}

Table 1 . The distribution of age and gender of patients by initial biopsy method.

The group of initial biopsy methods did not differ in terms of patients' age and gender ( $p=0.68$ and $\mathrm{p}=0.53$, respectively). Among 164 subjects, 104 (63.4\%) had malignant pleural disease, 15 (9.1\%) had tuberculous pleurisy, and $45(27.4 \%)$ had benign pleural disease at final diagnosis. The distribution of patients into the diagnostic arms and corresponding results are indicated in Figure 2. The overall diagnostic sensitivity, specificity, positive and negative predictive values (PPV and NPV), and positive and negative likelihood ratios (+LR and -LR) are shown in Table 2.

\section{Figure 2}

Figure 2. The distribution of performed pleural biopsy methods within the diagnostic workup and their corresponding results.

\section{$\underline{\text { Table } 2}$}

Table 2. Diagnostic sensitivity, specificity, positive and negative predictive values, and negative likelihood ratios according to the biopsy method and the entire work-up.

In 17 patients undergoing CT-ANPB, histopathological diagnosis was reported as fibrinous pleuritis. After discussing with patients, six underwent MT, four underwent VATS, and one underwent open surgical biopsy. The remaining six patients were followed up conservatively as they were unwilling to undergo further procedures. Four patients who underwent advanced invasive procedure and one patient who was monitored conservatively were eventually diagnosed 
with a malignant pleural disease (false-negative initial biopsy). Three had malignant pleural mesothelioma and two had metastatic malignant pleural involvement.

Among 35 patients who had fibrinous pleuritis on histopathological evaluation after MT, 31 had none of risk factors for malignant pleural disease that were pre-defined. After three years of close clinical monitoring, not a single case of malignancy was detected. Four patients underwent VATS due risk factors; three were diagnosed with malignant pleural mesothelioma, and the remaining patient had benign disease with no evidence of malignancy during 3-year's follow-up.

In the US-CNPB arm, two of 22 patients had fibrinous pleuritis histopathologically. One of these patients had risk factors on CT scan, and was diagnosed with malignant mesothelioma on open surgical biopsy. The other patient had no risk factors and 3-year follow-up revealed no evidence of malignant disease.

Five of 37 patients who had fibrinous pleuritis on MT or CPNB after initial biopsy procedures had risk factors for malignant diseases based on the pre-defined criteria. Malignant disease was determined by advanced invasive procedure in four of these subjects. There were no false negative cases in 32 patients who had no malignant disease risk factors.

Diagnostic sensitivity, specificity, PPV, NPV, -LR of the entire work-up for which efficacy and reliability was examined according to the pleural diseases are shown in Table 3.

\section{$\underline{\text { Table } 3}$}

Table 3. Diagnostic sensitivity, specificity, positive and negative predictive values, and negative likelihood ratios of the entire workup according to the pleural diseases.

In our study population, the number of patients with malignant pleural pathology was 104 . According to the proposed algorithm, 43 patients (41.3\%) underwent CT-ANPB, 41 patients (39.4\%) MT, and the rest (19.2\%) US-CNPB as initial procedure. The majority of patients (10/15; $66.7 \%$ ) with tuberculous pleurisy underwent CT-ANPB (Table 2).

There were 45 patients with benign pleural disease. Based on the proposed diagnostic work-up, 12 patients (26.7\%) underwent CT-ANPB, 32 patients (71.1\%) MT, and one patient (2.2\%) USCNPB as initial procedure. The percentage of performing MT was significantly higher than that of needle biopsy $(\chi 2=6.468 ; \mathrm{p}<0.011)$. 
Distribution of observed complications by biopsy method is shown in Table 4 .

\section{$\underline{\text { Table } 4}$}

Table 4. Complications in diagnostic arms.

A total of 65 patients in Arm A; 65 CT-ANPB, 6 MT, 4 VATS, and 1 open surgical biopsy were performed. Complications occurred in $11(14.4 \%)$ of the patients in a total of 76 procedures, all of them minor complications. One patient received paracetamol for analgesia, one patient received oral antibiotics for local infection. In Arm B a total of 77 patients were treated with 77 MT and 4 VATS. The number of patients experiencing complications in a total of 81 procedures was 14 (14/81: 17.2\%). One patient developed major complication, bleeding required 1 UI blood transfusion. A total of 22 patients in Arm C; 22 US-CNPB and 1 VATS were performed. In 23 patients, 3 patients (13.0\%) experienced complications. Hemoptysis, about 2-3 ml, did not require additional treatment.

\section{DISCUSSION}

With the increasing data on diagnostic biopsy strategies for pleural disease, there are a range of potential investigations open to clinicians in this common clinical scenario $(2,8-12,24-29)$. This is the first study which demonstrates the utility, high diagnostic yield and safety of a biopsy strategy based on triage of the initial imaging findings. The diagnostic sensitivity of the work-up which was evaluated in our study was $92.4 \%$. The diagnostic sensitivity of CT-ANPB was $90.6 \%$, MT was $93.3 \%$ and US-CNPB was $95.2 \%$ with $100 \%$ specificity. These figures are higher than that reported in the literature for the individual biopsy methods, and we hypothesize that that this is due to performing the appropriate biopsy method according to the initial CT findings. As such, this algorithm represents an integrated diagnostic work-up with high yield in a real life scenario, and we beleive our results can be used to alter the current diagnostic pathway.

In the literature, there exists no consensus regarding the best imaging method with which to guide pleural biopsies, as is the case regarding the type of needle used. Most studies have reported similarly high diagnostic accuracies for both techniques $(6,10,13,22,25-27)$. The choice between US and CT guidance is often influenced by factors such as individual preference, expertise, appliance availability and cost $(9,13,14,18)$. The reason for our choice of initial CT as the triage 
method is that it is usually performed and assessed in patients in whom pleural invasive procedure are considered.

In Arm A, we used Abrams' needle as biopsy needle as a study reported that it could obtain larger tissue samples in patients with tuberculous pleurisy (8), and it has higher diagnostic sensitivity in patients with limited pleural thickening $(8,9,28)$. This technique is also well known and widely used. The only difference in our methodology was to mark the most probable lesion area on the patients' skin, as determined by CT scan prior to biopsy $(9,10)$, which does not bear any additional cost to the standard application of Abrams' needle.

We found a high diagnostic sensitivity of $90.6 \%$ in 65 patients that we performed CT-ANPB. In a previous study, we compared CT-ANPB with US-CNPB and with MT, and found diagnostic sensitivities of $82.4 \%$, and $87.5 \%$, respectively $(9,10)$. In these studies, the procedure was applied to all patients that were randomized, i.e. also to those with only pleural effusion. In the present study, the techniques were used after patient selection post CT scan which might explain the higher diagnostic yields.

The patients assigned to the MT group included those with merely pleural effusion or those who had inaccessible lesion by Abrams' needle due to atypical pleural location or those with higher risk of BAP since most of metastatic malignant pleural pathologic conditions might manifest themselves only by pleural effusion on CT $(15,16)$. Many benign conditions except parapneumonic pleurisy require visualization of pleural space to establish definite diagnosis by ruling out malignant disease and tuberculosis $(3,29)$. No lesion except pleural fluid could be seen on CT scans of most of these patients $(15,16)$. In fact, diagnostic sensitivity in MT group that was performed in patients with just pleural effusion or suspected BAP was $93.3 \%$ in this study.

Diagnostic sensitivity of US-CNPB which we performed in patients with pleural thickening alone with no effusion in CT scan was detected to be as high as $95.2 \%$. This high ratio in this easy-toapply procedure arises from the fact that it was performed directly from pleural lesion/thickening area as in other studies $(11,13,22)$.

Correct diagnosis was achieved in 155 of 164 (94.5\%) patients after initial procedures in the algorithm. This was similar to the diagnostic sensitivity that would have been achieved if all patients had undergone MT $(3,10,29)$. We believe this high sensitivity is attributed to the fact that the decision about the type of biopsy that would be performed in selected patients on CT scan results had been already made. In fact, diagnostic accuracies were very close to each other in all diagnostic groups composed according to pleural effusion/lesion type (Table 2). 
A further important finding in our study was that the negative predictive values and negative likelihood ratios of the diagnostic work-up and biopsy methods were within reliable ranges (Table 2,3). It should be noted that the NPV of US-CNPB was based on only 2 patients (Figure 2). Previous reports have suggested that the negative predictive values and negative likelihood ratios of the needle biopsies are not reliable (30). In our two previous randomized trials, when the results are pooled according to the biopsy method used, the diagnostic sensitivity, specificity, positive and negative predictive value, and negative likelihood ratio are shown in Table $5(9,10)$. Patients were randomized to CT-ANPB versus MT, CT-ANPB versus US-CNPB, regardless of CT findings in these studies. Negative predictive values and negative likelihood ratios obtained in CTANPB and US-CNPB groups, as seen in the table, emphasize the necessity of a second procedure if the biopsy result is fibrinous pleuritis.

\section{$\underline{\text { Table } 5}$}

Table 5. Diagnostic sensitivity, specificity, positive and negative predictive values, and negative likelihood ratios according to the diagnostic methods in our previous series.

The rate of false negativity in the work-up after initial diagnostic procedure was determined as $5.4 \%(9 / 164)$.

We also used previously identified risk factors to reduce or eliminate false negative rate (23). When patients with a histopathological diagnosis of fibrinous pleuritis were assessed in terms of risk factors, five cases were detected to have risk factors, four of which (80\%) indicated malignant disease (Figure 1). There was only one patient with risk factor, yet was reported to have benign condition with VATS.

Needle biopsy was used in 87 of 164 (53.1\%) patients at first step. If its diagnostic accuracy had been low, the percentage of patients required to undergo a second, i.e. an invasive procedure would have been elevated, which in turn, led to increased costs and complications. In our proposed diagnostic work-up, most of patients with malignant pleural pathology received needle biopsy whereas most of those with benign pleural condition received MT as initial procedure. Therefore, not only the rate of performing MT, a more invasive and costly procedure, was reduced in malignant cases, but also needle biopsy, which was likely to be unnecessary, could be avoided in benign cases. Indeed, we had 45 cases with benign pleural disease except pleurisy tuberculosis. If 
the diagnostic procedures had been begun with needle biopsy, MT would be required as the second step since all these had fibrinous pleuritis.

Regarding complication rates, all methods proved safe among our patients like other publications regarding image-guided biopsies and MT have also reported low complication rates $(3,4,8$ $10,12,20,24)$.

In conclusion, we recommend that the proposed diagnostic workup in this study can be used as a diagnostic algorithm for pleural diseases which require histopathological analysis. In this algorithm, we suggest that CT-ANPB can be used as a first diagnostic evaluation after cytological investigation of the fluid in those cases where pleural thickness or pleural lesions are seen on CT. In patients with only fluid on CT, MT should be the first method in order to improve the chances of definite diagnosis. If benign asbestos pleurisy is suspected, the first method for diagnosis should preferentially be MT for the exclusion of malignancy. US-assisted CNPB can be used preferentially in cases in which only pleural thickening with no pleural effusion or a small pleural effusion is detected. Determination of risk factors predicting malignant disease in patients where fibrinous pleuritis is reported after MT would be useful for our clinical practice.

\section{REFERENCES}

1. Rahman NM, Gleeson FV. Image-guided pleural biopsy. Curr Opin Pulm Med. 2008; 14: 33136.

2. Koegelenberg CFN, Irusen EM, von Groote-Bidlingmaier F, Bruwer JW, Batubara EM, Diacon AH. The utility of ultrasound-guided thoracentesis and pleural biopsy in undiagnosed pleural exudates. Thorax. 2015; 70: 995-997.

3. Rahman NM, Ali NJ, Brown G, Chapman SJ, Davies RJ, Downer NJ, Gleeson FV, Howes TQ, Treasure T, Singh S, Phillips GD; BTS Pleural Disease Guideline Group. Local anaesthetic thoracoscopy. British Thoracic Society Pleural Disease Guideline 2010. Thorax. 2010; 65 (Suppl 2): ii54-60.

4. Dixon G, de Fonseka D, Maskell N. Pleural controversies: image guided biopsy vs. thoracoscopy for undiagnosed pleural effusions? J Thorac Dis. 2015; 6: 1041-51.

5. Hooper C, Lee YC, Maskell N; BTS Pleural Guideline Group. Investigation of a unilateral pleural effusion in adults. British Thoracic Society Pleural Disease Guideline 2010. Thorax. 2010; 65 (Supp1 2): ii4-17

6. Cao YY, Fan N, Xing F, Xu LY, Qu YJ, Liao MY. Computed tomography-guided cutting needle pleural biopsy: Accuracy and complications. Exp Ther Med. 2015; 9: 262-66. 
7. Wang J, Zhou X, Xie X, Tang Q, Shen P, Zeng Y. Combined ultrasound-guided cutting-needle biopsy and standard pleural biopsy for diagnosis of malignant pleural effusions. BMC Pulm Med. 2016; 16: 155-164.

8. Koegelenberg CFN, Bolliger CT, Theron J, Walzl G, Wright CA, Louw M, Diacon AH. Direct comparison of the diagnostic yield of ultrasound-assisted Abrams and Tru-Cut needle biopsies for pleural tuberculosis. Thorax. 2010; 65: 857-62

9. Metintas M, Yildirim H, Kaya T, Ak G, Dundar E, Ozkan R, Metintas S. CT Scan-Guided Abrams' Needle Pleural Biopsy versus Ultrasound-Assisted Cutting Needle Pleural Biopsy for Diagnosis in Patients with Pleural Effusion: A Randomized, Controlled Trial. Respiration. 2016; 91: 156-63.

10. Metintas M, Ak G, Dundar E, Yildirim H, Ozkan R, Kurt E, Erginel S, Alatas F, Metintas S. Medical thoracoscopy vs CT scan-guided Abrams pleural needle biopsy for diagnosis of patients with pleural effusions: a randomized, controlled trial. Chest. 2010; 137: 1362-68

11. Stigt JA, Boers JE, Groen HJ. Analysis of "dry" mesothelioma with ultrasound guided biopsies. Lung Cancer. 2012; 78: 229-33.

12. Koegelenberg CF, Diacon AH. Pleural controversy: close needle pleural biopsy or thoracoscopy-which first ? Respirology. 2011; 16: 738-46..

13. Koegelenberg CFN, Diacon AH. Image-guided pleural biopsy. Curr Opin Pulm Med. 2013; 19: 368-73.

14. Matin TN, Gleeson FV. Interventional radiology of pleural diseases. Respirology. 2011; 16: 419-29.

15. Leung AN, Müller NL, Miller RR. CT in differential diagnosis of diffuse pleural disease. AJR Am J Roentgenol. 1990; 154: 487-92.

16. Metintas M, Ucgun I, Elbek O, Yildirim H, Ozkan R, Kurt E, Erginel S, Alatas F, Metintas S. Computed tomography features in malignant pleural mesothelioma and other commonly seen pleural diseases. Eur J Radiol. 2002; 41: 1-9.

17. Samirashojaee S, Lee HJ. Thoracoscopy: medical versus surgical-in the management of pleural diseases. J Thorac Dis. 2015; 7: S339-351

18. Hallifax RJ, Corcoran JP, Ahmed A, Nagendran M, Rostom H, Hassan N, Maruthappu M, Psallidas I, Manuel A, Gleeson FV, Rahman NM. Physician-based ultrasound-guided biopsy for diagnosing pleural disease. Chest. 2014; 146: 1001-06.

19. Maskell NA, Gleeson FV, Davies RJ. Standard pleural biopsy versus CT-guided cuttingneedle biopsy for diagnosis of malignant disease in pleural effusions: a randomised controlled trial. Lancet. 2003; 19; 361:1326-30. 
20. Benamore RE, Scott K, Richards CJ, Entwisle JJ. Image-guided pleural biopsy: diagnostic yield and complications. Clin Radiol. 2006; 61: 700-05.

21. Koegelenberg CFN, von Groote-Bidlingmaier F, Bolliger CT. Transthoracic ultrasonography for the respiratory phsyician. Respiration. 2012, 84: 337-50

22. Stigt JA, Groen HJ. Percutaneous ultrasonography as imaging modality and sampling guide for pulmonologists. Respiration. 2014; 87: 441-51.

23. Metintas M, Ak G, Cadirci O, Yildirim H, Dundar E, Metintas S. Outcome of patients diagnosed with fibrinous pleuritis after medical thoracoscopy. Respir Med. 2012; 106: 1177 83.

24. Qureshi NR, Rahman NM, Gleeson FV. Thoracic ultrasound in the diagnosis of malignant pleural effusion. Thorax. 2009; 64: 139-43.

25. Metintaş M, Özdemir N, Işıksoy S, Kaya T, Ekici M, Erginel S, Harmancı E, Erdinç P, Ülgey $\mathrm{N}$, Alataş F. CT-guided pleural needle biopsy in the diagnosis of malignant mesothelioma. $\mathrm{J}$ Comput Assist Tomog. 1995;19: 370 - 374

26. Adams RF, Gleeson FV. Percutaneous image-guided cutting-needle biopsy of the pleura in the presence of a suspected malignant effusion. Radiology. 2001; 219: 510-14.

27. Bibby AC, Maskell NA. Pleural biopsies in undiagnosed pleural effusions; Abrams vs imageguided vs thoracoscopic biopsies. Curr Opin Pulm Med. 2016; 22: 392-98.

28. Botana-Rial M, Leiro-Fernández V, Represas-Represas C, González-Piñeiro A, Tilve-Gómez A, Fernández-Villar A. Thoracic ultrasound-assisted selection for pleural biopsy with Abrams needle. Respir Care. 2013; 58: 1949-54.

29. Casal RF, Eapen GA, Morice RC, Jimenez CA. Medical thoracoscopy. Curr Opin Pulm Med. 2009; 15: 313-20.

30. Báez-Saldaña R, Rumbo-Nava U, Escobar-Rojas A, Castillo-González P, León-Dueñas S, Aguirre-Pérez T, Vázquez-Manríquez ME. Accuracy of closed pleural biopsy in the diagnosis of malignant pleural effusion. J Bras Pneumol. 2017; 43: 424-30. 
Table 1. The distribution of age and gender of patients by initial biopsy method.

\begin{tabular}{|l|l|l|l|l|}
\hline \multirow{2}{*}{$\begin{array}{l}\text { Patient } \\
\text { characteristics }\end{array}$} & \multicolumn{4}{|l|}{ Biopsy methods } \\
\cline { 2 - 5 } & CT-ANPB & MT & US-CNPB & Total \\
\hline $\begin{array}{l}\text { Mean age, yrs, } \\
\text { (range) }\end{array}$ & $59.5(19-82)$ & $63.7(35-89)$ & $65.8(42-83)$ & $62.3(19-89)$ \\
\hline Gender; male/female & $42 / 35$ & $34 / 31$ & $9 / 13$ & $85 / 79$ \\
\hline
\end{tabular}


Table 2. Diagnostic sensitivity, specificity, positive and negative predictive values, and negative likelihood ratios according to the biopsy method and the entire work-up.

\begin{tabular}{|l|c|c|c|c|}
\hline \multirow{2}{*}{ Ratios } & \multicolumn{4}{|c|}{ Biopsy methods } \\
\cline { 2 - 5 } & CT-ANPB & MT & US-CNPB & Work-up \\
\hline Sensitivity & $90.6 \%$ & $93.3 \%$ & $95.2 \%$ & $92.4 \%$ \\
95\% CI & $(79.3-96.9)$ & $(81.7-99.0)$ & $(76.2-99.9)$ & $(86.1-96.5)$ \\
\hline $\begin{array}{l}\text { Specificity } \\
\text { 95\% CI }\end{array}$ & $100 \%$ & $100 \%$ & $100 \%$ & $100 \%$ \\
\hline $\begin{array}{l}\text { Positive predictive value } \\
\text { 95\% CI }\end{array}$ & $173.5-100.0)$ & $(89.1-100.0)$ & $(2.5-100.0)$ & $(92.1-100.0)$ \\
\hline $\begin{array}{l}\text { Negative predictive value } \\
\text { 95\% CI }\end{array}$ & $70.6 \%$ & $100 \%$ & $100 \%$ & $100 \%$ \\
\hline $\begin{array}{l}\text { Negative likelihood ratio } \\
\text { 95\% CI }\end{array}$ & $(51.0-84.7)$ & $(78.1-97.0)$ & $(12.9-87.1)$ & $(72.7-90.4)$ \\
\hline $\begin{array}{l}\text { Accuracy } \\
\text { 95\% CI }\end{array}$ & 0.09 & 0.07 & 0.05 & 0.08 \\
\hline
\end{tabular}

Positive likelihood ratio was not estimated because specificities and PPVs $100 \%$ for all methods. 
Table 3. Diagnostic sensitivity, specificity, positive and negative predictive values, and negative likelihood ratios of the entire workup according to the pleural diseases.

\begin{tabular}{|l|c|c|c|}
\hline \multicolumn{1}{|c|}{ Algorithm } & \multicolumn{3}{|c|}{ Pleural diseases } \\
\cline { 2 - 4 } & $\begin{array}{c}\text { Malignant pleural } \\
\text { mesothelioma }\end{array}$ & $\begin{array}{c}\text { Metastatic malignant } \\
\text { pleural diseases }\end{array}$ & $\begin{array}{c}\text { Tuberculous } \\
\text { pleurisy }\end{array}$ \\
\hline $\begin{array}{l}\text { Sensitivity } \\
\text { 95\% CI }\end{array}$ & $\begin{array}{c}86.8 \% \\
(74.6-94.5)\end{array}$ & $\begin{array}{c}96.1 \% \\
(86.5-99.5)\end{array}$ & $\begin{array}{c}100 \% \\
(78.2-100.0)\end{array}$ \\
\hline $\begin{array}{l}\text { Specificity } \\
\text { 95\% CI }\end{array}$ & $100 \%$ & $100 \%$ & $100 \%$ \\
$(94.6-100.0)$ & $(94.7-100.0)$ & $106.5-100.0)$ \\
\hline $\begin{array}{l}\text { Positive predictive value } \\
\text { 95\% CI }\end{array}$ & $100 \%$ & $100 \%$ & $100 \%$ \\
\hline $\begin{array}{l}\text { Negative predictive value } \\
\text { 95\% CI }\end{array}$ & $90.4 \%$ & $97.1 \%$ & 0.00 \\
\hline $\begin{array}{l}\text { Negative likelihood ratio } \\
\text { 95\% CI }\end{array}$ & $(82.5-95.0)$ & $(89.7-99.3)$ & 0.04 \\
\hline $\begin{array}{l}\text { Accuracy } \\
\text { 95\% CI }\end{array}$ & $(0.07-0.26)$ & $(0.01-0.15)$ & $100 \%$ \\
\hline
\end{tabular}

Positive likelihood ratio was not estimated because specificities and PPVs $100 \%$ for all methods. 
Table 4. Complications in diagnostic arms.

\begin{tabular}{|l|c|c|c|}
\hline \multicolumn{1}{|c|}{ Side effect } & $\begin{array}{c}\text { CT-ANPB } \\
\mathbf{n , ~ ( \% )}\end{array}$ & $\begin{array}{c}\text { MT } \\
\mathbf{n , ( \% )}\end{array}$ & $\begin{array}{c}\text { US-CNPB } \\
\mathbf{n , ~ ( \% )}\end{array}$ \\
\hline Number of biopsies & 76 & 81 & 23 \\
\hline Major & - & $1(1.2)$ & - \\
Bleeding & - & - & $1(4.3)$ \\
Hemoptysis & $1(1.3)$ & $1(1.2)$ & - \\
\hline Minor & $1(1.3)$ & $1(1.2)$ & - \\
Bleeding (less than 100 ml) & $2(2.6)$ & $1(1.2)$ & - \\
Hypotansion & - & $1(1.2)^{*}$ & - \\
Syncope & $3(3.9)$ & $9(11.1)$ & - \\
Extended air leakage (more than 3 days) & $2(2.6)$ & $1(1.2)$ & $1(4.3)$ \\
Subcutanous emphysema ${ }^{\mu}$ & - & $2(2.4)$ & - \\
Pain & $1(1.3)$ & - & $1(4.3)$ \\
Tachycardia & $1(1.3)$ & $1(1.2)$ & - \\
Fever & $2(2.6)$ & $1(1.2)$ & - \\
Nause or vomiting & $1(1.3)$ & $1(1.2)$ & $1(4.3)$ \\
Percutaneous edema** & & \\
Wound infection & & & \\
\hline
\end{tabular}

*Spontaneously resolved on day $4 .{ }^{* *}$ The amount of the leakage was palpable by a palm. 
Table 5. Diagnostic sensitivity, specificity, positive and negative predictive values, and negative likelihood ratios according to the diagnostic methods in our previous series.

\begin{tabular}{|l|c|c|c|}
\hline \multirow{2}{*}{ Ratios } & \multicolumn{3}{|c|}{ Biopsy methods } \\
\cline { 2 - 4 } & CT-ANPB & MT & US-CNPB \\
\hline Sensitivity & $83.5 \%$ & $94.1 \%$ & $61.8 \%$ \\
95\% CI & $(74.9-90.1)$ & $(83.8-98.8)$ & $(47.7-74.6)$ \\
\hline Specificity & $100 \%$ & $100 \%$ & $100 \%$ \\
95\% CI & $(89.1-100.0)$ & $(71.5-100.0)$ & $(80.5-100.0)$ \\
\hline Positive predictive value & $100 \%$ & $100 \%$ & $100 \%$ \\
95\% CI & & & \\
\hline Negative predictive value & $65.3 \%$ & $78.6 \%$ & $44.7 \%$ \\
95\% CI & $(54.9-74.4)$ & $(55.0-91.7)$ & $(36.6-53.1)$ \\
\hline Negative likelihood ratio & 0.17 & 0.06 & 0.38 \\
95\% CI & $(0.11-0.25)$ & $(0.02-0.18)$ & $0.27-0.53$ \\
\hline Accuracy & $87.4 \%$ & $95.2 \%$ & $70.8 \%$ \\
95\% CI & $(80.6-92.5)$ & $(86.5-99.0)$ & $(58.9-81.0)$ \\
\hline
\end{tabular}

Positive likelihood ratio was not estimated because specificities and PPVs $100 \%$ for all methods. 